\title{
The effects of a pro-active integrated care intervention for frail community-dwelling older people: a quasi-experimental study with the GP-practice as single entry point
}

Wilhelmina Mijntje Looman*, Isabelle Natalina Fabbricotti, Ruben de Kuyper and Robbert Huijsman

\begin{abstract}
Background: This study explored the effectiveness of a pro-active, integrated care model for community-dwelling frail older people compared to care as usual by evaluating the effects on a comprehensive set of outcomes: health outcomes (experienced health, mental health and social functioning); functional abilities; and quality of life (general, health-related and well-being).

Methods: The design of this study was quasi-experimental. In this study, 184 frail older patients of three GP practices that implemented the Walcheren Integrated Care Model were compared with 193 frail older patients of five GP practices that provided care as usual. In the Walcheren Integrated Care Model, community-dwelling elderly were pro-actively screened for frailty from the GP practice using the Groningen Frailty Indicator, and care needs were assessed with the EASYcare instrument. The GP practice functioned as single entry point from which case management was provided, and the GP was the coordinator of care. The entire process was supported by multidisciplinary meetings, multidisciplinary protocols and web-based patient files. The outcomes of this study were obtained at baseline, after 3 months and after 12 months and analyzed with linear mixed models of repeated measures.
\end{abstract}

Results: The Walcheren Integrated Care Model had a positive effect on love and friendship and a moderately positive effect on general quality of life. The ability to receive love and friendship and general quality of life decreased in the control group but was preserved in the experimental group. No significant differences were found on health outcomes such as experienced health, mental health, social functioning and functional abilities.

Conclusions: The results indicated that pro-active, integrated care can be beneficial for frail older people in terms of quality of life and love and friendship but not in terms of health outcomes and functional abilities. Recommendations for future research are to gain greater insight into what specific outcomes can be achieved with proactive and integrated care, considering the specific content of this care, and to allow for the heterogeneity of frail older people in evaluation research.

Trial registration: Current Controlled Trials ISRCTN05748494. Registration date: 14/03/2013.

Keywords: Integrated care, Prevention, Frail older people, Effectiveness, Outcome

\footnotetext{
* Correspondence: looman@bmg.eur.nl

Erasmus University Rotterdam, Institute of Health Policy and Management, P.O. Box 1738, 3000 DR, Rotterdam, The Netherlands
} 


\section{Background}

The care for community-dwelling frail older people poses a real challenge for health care systems. Due to population ageing, the number of frail older people is increasing rapidly [1]. Furthermore, national health policies are aimed at preventing admission to nursing homes because institutionalization is costly. Frail older people themselves prefer to grow old in the community [2] and want to live independently at home as long as possible; also referred to as 'ageing in place' [3]. This could become problematic because frail older people suffer from problems in the physical, psychological and social domains of daily functioning [4]. The quality of care for these frail older people living in the community needs improvement [5]. Currently, care is reactive and the needs of frail older people are not addressed in a timely manner, leading to crisis situations [6]. Care is also fragmented and lacks continuity and coordination [7]. As a way to mitigate these challenges, care for frail older patients in the community should become more pro-active and integrated [8].

Pro-active care for frail older people starts with the identification of this group within the community. Research has shown that frailty is related to negative health outcomes, disability [9], and poor quality of life [10]. To postpone or prevent these outcomes, frailty should be identified quickly and correctly [11, 12]. After the proactive identification, care should be integrated and delivered coherently according to the needs of the frail individuals related to the areas of prevention, care, cure, housing and welfare [13], meaning that professionals from different disciplines and sectors should collaborate $[14,15]$. In the present study, we evaluated the Walcheren Integrated Care Model (WICM), a specific proactive and integrated care intervention aimed at community-dwelling frail older people and implemented in primary care with the GP practice as single entry point and the GP as coordinator of care. This study contributes to the growing body of evidence due to the specific features of the intervention and its extensive evaluation.

WICM is primarily characterized by the combination of a pro-active and integrated approach to care for frail community-dwelling patients. Many care interventions for community-dwelling frail older people have a strong focus on integration, but the importance of proactiveness is not widely acknowledged. In the WICM, frailty is detected from the GP practice by screening the GP's entire patient population aged 75 years and older. Research has shown that such a pro-active approach, in combination with integrated care elements, is more effective than a pro-active approach alone [16]. Moreover, all integrated care elements that have been recognized to be effective in prior research are included in the
WICM instead of considering only a selection of these elements. These elements include the following: geriatric assessments, case management, multidisciplinary teams, a single entry point [17], multidisciplinary protocols and discussions, web-based patient files, and a network structure $[15,18,19]$. This network structure, in which the WICM is embedded, consists of GP practices, home care organizations, nursing homes and patient organizations. The representatives of these involved organizations form the WICM's Steering Committee, which is an example of organizational integration at the meso-level. This organizational integration is also a specific feature of the WICM because most integrated care interventions are characterized by case management and the relationship between the GP and case manager [20], and integration is restricted to the micro level. The assumption for our approach is that adopting more strategies at different levels is essential to achieve effectiveness [21].

The effectiveness of the pro-active and integrated WICM is evaluated comprehensively by considering an extensive combination of patient outcome measures. Previous evaluation studies have primarily focused on three categories of outcomes corresponding to the three problem areas of frailty: health outcomes, functional abilities and quality of life [16, 22-36]. However, these studies have shown inconsistent results and there is an urgent need for more in-depth evaluation research, in particular for research reporting these three domains simultaneously [25]. Even though no intervention established effects in terms of health outcomes, functional abilities and quality of life yet [16, 24], we intended to explore whether the pro-active, comprehensive and highly integrated WICM can achieve effectiveness in all three categories. Hence, this study aimed to answer the following research question: what is the effect of the WICM on health outcomes (experienced health, mental health, social functioning), functional abilities and quality of life (general, health-related, and well-being) of community-dwelling frail older people?

\section{Methods \\ Design}

The design of this study was quasi-experimental and included before and after measurements with a control group (see also [37]). The measurements were obtained at baseline, after 3 months and after 12 months. The experimental group consisted of older patients of eight GPs from three GP practices located in eastern Walcheren who provided care according to the WICM. The control group consisted of the patients of six GPs from five GP practices who provided care as usual in the northern, southern and western parts of Walcheren.

The study design was reviewed by the medical ethics committee of the Erasmus Medical Center, Rotterdam, 
the Netherlands, under protocol number MEC-2013058. This committee waived further examination because the rules established in the Medical Research Involving Human Subjects Act did not apply.

\section{Participants}

All GP patients aged 75 and older of the 3 GP practices in the experimental $(n=892)$ and 6 GP practices in the control group $(n=953)$ were sent a GFI questionnaire and an informed consent (see Fig. 1). The GFI is a 15item questionnaire screening for frailty that measures decreases in physical, cognitive, social and psychological functioning. GFI scores range from 0 to 15; patients with a score of 4 or higher were considered frail $[38,39]$. In the experimental region $83 \%$ of the patients returned the GFI questionnaire; in the control region $78 \%$. Patients were included in the study when they did not fulfil the exclusion criteria of not being frail (GFI score lower than 4); living in a nursing home; being on waiting list for a nursing home; and being terminally ill with a life expectancy under 6 months. At baseline, 254 frail older patients were included in the experimental group, and 249 frail older patients were included in the control group. After 12 months, the final study population included 184 frail older people in the experimental group and 193 frail older people in the control group. Loss to follow-up was mostly caused by frail older people refusing to participate $(n=54)$ or passing away $(n=23)$.

\section{Intervention}

After screening the patient population of each GP with the GFI, frail older patients in the experimental group were visited by a nurse practitioner who assessed their functional, cognitive, mental and psychological functioning using EASYcare. EASYcare is an evidence-based, comprehensive instrument used to assess care needs [31] and has a separate model to translate care needs into specific treatments goals. The GP and nurse practitioner decided on treatment goals in consultation with the older people and their informal caregivers, which

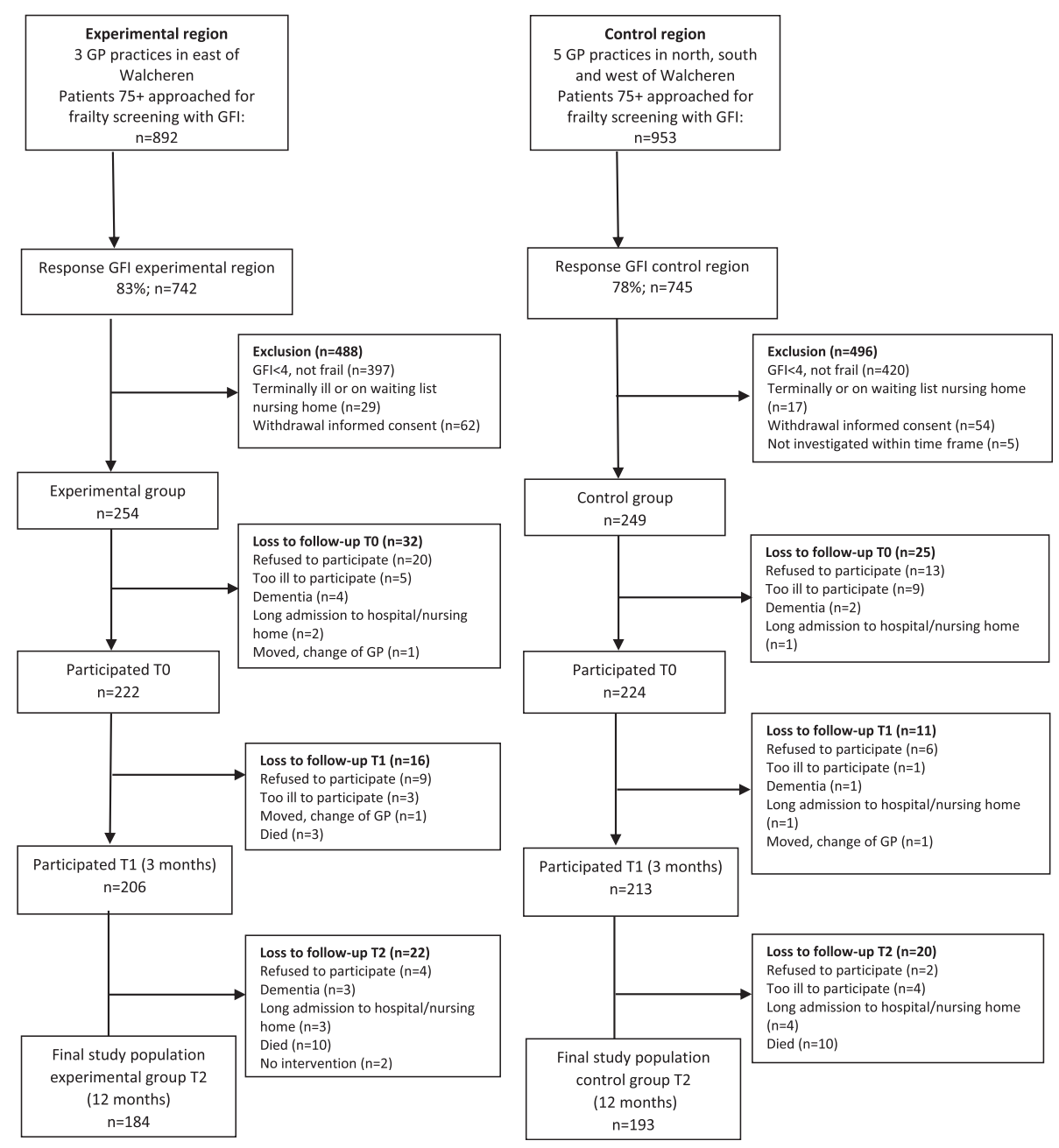

Fig. 1 Flow chart of participants 
were translated into a preliminary multidisciplinary treatment plan. This plan was determined in a multi-disciplinary meeting attended by at least the GP, the nurse practitioner, and a secondary-line geriatric nurse practitioner. Depending on frail elderly's problems discussed, the meeting was also attended by other health professionals such as geriatric physiotherapists, geriatricians, pharmacists, district nurse, nursing home doctors and mental health workers. The concrete actions, activities and responsibilities of these health professionals were discussed during this meeting.

Case management was provided from the GP-practice by the nurse practitioner or by a secondary-line geriatric nursing practitioner, depending on the complexity of the older people person's problems. The case manager coordinated care within the multidisciplinary team which implies monitoring the frail older person's condition, arranging the admittance to the required services, being the contact person for the involved professionals to coordinate their care and periodically evaluating the multidisciplinary treatment plan. The evaluation occurred in multidisciplinary meetings. The entire process was supported by web-based patient files and multidisciplinary protocols describing the responsibilities and activities of the involved professionals, in particular the nurse practitioner and secondary-line geriatric nursing practitioner who provided case management. Protocols were also designed for common themes such as incontinence, polypharmacy, and falling. In the WICM, the GP had the final responsibility and functioned as a coordinator of care and partner in prevention. The GP practice was a single entry point for the older frail patients, their informal caregivers and health professionals where they could gain access to information and services of all involved professionals and organizations.

The model required task reassignment and delegation between nurses and doctors and among GPs, nursing home doctors and geriatricians. Consultations among primary, secondary, and tertiary care providers occurred. Regarding integration at the organizational level, a
Steering Committee serves as an umbrella organization under which the WICM is developed and disseminated. The Steering Committee consists of representatives from all involved organizations, such as GP practices, home care organizations and nursing homes, and provides the necessary provider network. Patient representatives support the project, and the health insurer $\mathrm{CZ}$ provides financial support for the project.

\section{Care as usual}

Compared with the WICM, care as usual in the Netherlands is reactive and fragmented (Table 1). Every Dutch citizen is registered at a particular GP practice (or family doctor) near their home. Dutch patients first consult their GP for all health problems. GPs play the role of gate keeper [40], patients must have a referral from their GP to obtain care from the primary, secondary and tertiary echelons [13]. However, patients solely receive care for specific (health) problems on their own initiative.

Care as usual is fragmented and has a mono-disciplinary focus. Even though the GP is a generalist and has the role of gatekeeper, communication between professionals from the different disciplines and sectors is bi-lateral through referral letters and sporadic telephone calls.

The GPs in the control group were unable to implement elements of the integrated model during the study period because they did not receive financial support from the health insurer to implement the integrated care activities of the WICM. Furthermore, the GPs in the control group could not treat frail older patients differently, as these GPs were not given information on who participated in the study. Therefore, the probability of bias was minimized [41].

\section{Data collection and measures}

Data was collected with questionnaires at three points in time: at baseline, after 3 months and after 12 months. All older people were visited at home by trained interviewers recruited from the region of Walcheren to

Table 1 Differences between WICM and care as usual

\begin{tabular}{|c|c|c|}
\hline & WICM & Care as usual \\
\hline Role GP & Single entry point, coordinator of care & Gatekeeper \\
\hline \multirow[t]{2}{*}{ Pro-activeness versus reactiveness } & Entire patient population of $75+$ is screened for frailty & Patients receive care on their own initiative \\
\hline & Comprehensive assessment of care needs with EASYcare & Patients receive care for specific health problems \\
\hline Treatment plan & Multidisciplinary treatment plan & No or monodisciplinary treatment plan \\
\hline Care coordination & $\begin{array}{l}\text { Case management: monitoring, admittance to services, } \\
\text { contact person for professionals, evaluating treatment plan }\end{array}$ & No case management \\
\hline Communication & Multidisciplinary meetings and web-based files & Bilateral communication by phone calls and letters \\
\hline Protocols & Multidisciplinary protocols & Monodisciplinary protocols \\
\hline Network & Network structure & No participation in provider network \\
\hline
\end{tabular}


ensure a cultural fit with the frail older people. Interviewers had a background in healthcare to ensure a high-quality interview.

Health, functional abilities and quality of life were studied, primarily with validated instruments. All health outcomes (experienced health, mental health and social functioning) were assessed by means of questions from the RAND-36 questionnaire [42]. Experienced health was assessed with one item from the RAND-36 that allows frail older people to evaluate their own health. Mental health was measured using a five-item RAND-36 scale with items that question how often the respondent feels certain emotions, such as happiness or nervousness; the Cronbach's alpha for this scale was 0.74. Social functioning was measured with one item that asked how often social activities were hampered by physical health or emotional problems.

Functional abilities were measured with the Katz-15 instrument that assesses the ability to perform 15 activities of daily living, such as getting dressed, shopping and taking medication [43]; the Cronbach's alpha of this instrument was 0.86 .

To assess quality of life, various instruments were used. First, a general measure of quality of life was used, which was based on the RAND-36 [42]. The second measure was the EQ-5D, which focuses on healthrelated quality of life and includes five dimensions: mobility, self-care, daily activities, pain/discomfort and mood $[44,45]$. The third measure was the ICECAP, which was specifically developed to assess the quality of life related to older people well-being. The ICECAP measures five dimensions of quality of life: attachment, security, role, enjoyment and control [46]. This instrument is based on Sen's capability approach, which focuses on whether older people are able to function within these domains [47]. All outcome variables are continuous and measured at the interval level.

The covariates included are age, gender, marital status (0: married and living together; 1 : single and widowed), living arrangement (0: independently; 1 : assisted living facility) and educational level (0: low; 1: high). Age is a continuous variable measured at the ratio level and all other covariates are categorical variables measured at the nominal level.

\section{Statistical analysis}

The study population was described, and baseline differences between the experimental and control groups were tested using chi square tests for categorical variables and independent t-tests for continuous variables. Each outcome variable after 3 and 12 months of follow-up was analyzed with linear mixed models of repeated measures. In all models, time and intervention (experimental and control group) were included and we adjusted for the baseline score of the specific outcome variable and for the covariates sex, age, marital status, educational level, and living arrangement. The significance level was set at $p<0.05$ and $\mathrm{p}$-values of $<0.10$ were also reported [48]. All analyses were performed with SPSS 22 .

\section{Results}

The study population consisted of frail older patients with an average age of 82 years and an average score of 6 on the GFI (see Table 2). Women were overrepresented in both groups: $70 \%$ of the experimental group and $60 \%$ of the control group were female. Sixty-three percent of the frail older people in the experimental group and $47 \%$ in the control group had a lower level of education. The majority of the frail older people did not have a partner and lived independently. Frail older people reported on average four morbidities; most common were joint damage, hearing problems, vision disorders and heart failure.

Compared with the control group, the experimental group consisted of significantly more women, more lesseducated individuals and more individuals residing in assisted living facilities.

The results at baseline showed that frail older people find their mental health and social functioning to be less problematic than their health. The average score on functional abilities was approximately 4 , meaning that frail older people need help with 4 (instrumental) activities of daily life. The score for health-related quality of life was approximately 0.6 , and the scores on the domains of well-being ranged from 2.6 to 3.2. At baseline, health outcomes, functional abilities and quality of life were equal in both groups, except for general quality of life. General quality of life was significantly lower at baseline in the experimental group than in the control group (42.3 vs. $47.0, p<0.05$ ).

Table 3 shows that the WICM had limited effects on health outcomes, functional abilities and quality of life. The WICM had a moderate significant effect on quality of life after 12 months (CI: $(-0.15$ to $5.63 ; p<0.10)$. Whereas the general quality of life of the frail older people in the control group decreased over 12 months, the quality of life of the frail older people in the experimental group was preserved. With regards to healthrelated quality of life and well-being, no effects were found. However, WICM impacted one dimension of well-being: the ability to receive love and friendship (CI: (0.14 to $0.36 ; p<0.001)$. In the control group, the ability to receive love and friendship decreased, but this ability did not change in the experimental group. No significant differences were found between the groups in terms of experienced health, mental health and social functioning. Moreover, functional abilities of frail older people were not affected by the WICM. 
Table 2 Baseline characteristics of the study population

\begin{tabular}{|c|c|c|c|}
\hline & $\begin{array}{l}\text { Experimental group }(n=184) \\
\text { Mean }(S D) \text { or } \%\end{array}$ & $\begin{array}{l}\text { Control group }(n=193) \\
\text { Mean (SD) or } \%\end{array}$ & p-value \\
\hline \multicolumn{4}{|l|}{ Background variables } \\
\hline GFI (0-15) & $6.0(2.0)$ & $5.8(1.8)$ & 0.19 \\
\hline Age & $81.8(4.7)$ & $82.3(5.3)$ & 0.38 \\
\hline Sex-women & $69.6 \%$ & $59.6 \%$ & 0.04 \\
\hline $\begin{array}{l}\text { Educational level } \\
\text { Low } \\
\text { High }\end{array}$ & $\begin{array}{l}63.0 \% \\
37.0 \%\end{array}$ & $\begin{array}{l}46.6 \% \\
53.4 \%\end{array}$ & 0.00 \\
\hline $\begin{array}{l}\text { Marital status } \\
\text { Married and living together } \\
\text { Single and widowed }\end{array}$ & $\begin{array}{l}37.0 \% \\
63.0 \%\end{array}$ & $\begin{array}{l}41.7 \% \\
58.3 \%\end{array}$ & 0.35 \\
\hline $\begin{array}{l}\text { Living situation } \\
\text { Independently } \\
\text { Assisted living facility }\end{array}$ & $\begin{array}{l}71.7 \% \\
28.3 \%\end{array}$ & $\begin{array}{l}82.4 \% \\
17.6 \%\end{array}$ & 0.01 \\
\hline Multimorbidity & $3.8(1.9)$ & $3.9(1.9)$ & 0.66 \\
\hline \multicolumn{4}{|l|}{ Outcomes } \\
\hline \multicolumn{4}{|l|}{ Health outcomes } \\
\hline Experienced health (0-100) & $33.8(17.1)$ & $35.1(20.5)$ & 0.51 \\
\hline Mental health (0-100) & $71.3(17.6)$ & $72.0(16.5)$ & 0.69 \\
\hline Social functioning (0-100) & $69.1(33.7)$ & $65.7(39.0)$ & 0.36 \\
\hline \multicolumn{4}{|l|}{ Functional abilities } \\
\hline Functional abilities (0-15) & $3.9(3.1)$ & $3.7(3.2)$ & 0.48 \\
\hline \multicolumn{4}{|l|}{ Quality of life } \\
\hline General quality of life $(0-100)$ & $42.3(18.0)$ & $47.0(19.4)$ & 0.01 \\
\hline Health-related quality of life $(0-1)$ & $0.6(0.2)$ & $0.7(0.3)$ & 0.60 \\
\hline Well-being - love \& friendship (1-4) & $3.1(0.8)$ & $3.0(0.8)$ & 0.20 \\
\hline Well-being - security (1-4) & $3.2(0.9)$ & $3.3(0.8)$ & 0.32 \\
\hline Well-being - role (1-4) & $2.7(0.8)$ & $2.8(0.8)$ & 0.12 \\
\hline Well-being - enjoyment (1-4) & $3.0(0.8)$ & $2.8(0.8)$ & 0.08 \\
\hline Well-being - control (1-4) & $2.6(0.9)$ & $2.8(0.9)$ & 0.08 \\
\hline
\end{tabular}

All baseline scores were strongly significant and were the main determinant for all outcomes after 12 months. Of the covariates, age was the most important and had a negative effect on social functioning, functional abilities, and health-related quality of life. Marital status had a negative effect on two outcomes, as frail older people with a partner showed lower scores for social functioning and functional abilities. In addition, two significant trends over time could be observed: functional abilities and health-related quality of life both decreased over time.

\section{Discussion}

In this study, we explored the effectiveness of the WICM in terms of health outcomes, functional abilities and quality of life. The WICM is an intervention that combines a pro-active and integrated care approach organized from the GP practice; the model contains diverse effective integrated care elements, and integration is achieved at the organizational level. Our study shows that the WICM has a positive effect on the ability to receive love and friendship, and the WICM moderately preserves the general quality of life of frail older people. The WICM is not effective in terms of health outcomes and functional abilities.

The effect of the WICM on quality of life could possibly be explained by the pro-active approach of the WICM and its target group. Previous research has shown that a pro-active attitude has positive results on quality of life [10] and that timely identification of frailty prevents further deterioration $[11,12]$. Moreover, in the WICM, older people are pro-actively screened for frailty from the GP practice with the GFI questionnaire, which strongly determined the target group for the intervention. The GFI questionnaire was sent to all GP patients aged 75 years or older and focuses on physical, cognitive, 
Table 3 Linear mixed models - adjusted overall effects ${ }^{a}$

\begin{tabular}{|c|c|c|c|c|}
\hline & Mean (SE) experimental & Mean (SE) control & Mean diff $(95 \% \mathrm{Cl})$ & p-value \\
\hline \multicolumn{5}{|l|}{ Outcomes } \\
\hline \multicolumn{5}{|l|}{ Health outcomes } \\
\hline Experienced health (0-100) & $34.31(1.01)$ & $34.99(1.04)$ & $-0.68(-3.18$ to 1.82$)$ & 0.59 \\
\hline Mental health (0-100) & $68.86(0.94)$ & $69.44(0.91)$ & $-0.42(-2.69$ to 1.85$)$ & 0.72 \\
\hline Social functioning (0-100) & $65.06(2.29)$ & $66.42(2.36)$ & $-1.36(-7.04$ to 4.33$)$ & 0.64 \\
\hline \multicolumn{5}{|l|}{ Functional abilities } \\
\hline Functional abilities (0-15) & $4.41(0.14)$ & $4.19(0.14)$ & $0.22(-0.13$ to 0.56$)$ & 0.21 \\
\hline \multicolumn{5}{|l|}{ Quality of life } \\
\hline General quality of life $(0-100)$ & $42.66(1.15)$ & $39.92(1.19)$ & $2.74(-0.15$ to 5.63$)$ & 0.06 \\
\hline Health-related quality of life (0-1) & $0.66(0.01)$ & $0.65(0.02)$ & 0.01 ( -0.03 to 0.04$)$ & 0.73 \\
\hline Well-being - love \& friendship (1-4) & $3.00(0.04)$ & $2.75(0.05)$ & 0.25 (0.14 to 0.36$)$ & 0.00 \\
\hline Well-being - security (1-4) & $3.32(0.05)$ & $3.28(0.06)$ & 0.05 (-0.08 to 0.18$)$ & 0.45 \\
\hline Well-being - role (1-4) & $2.57(0.05)$ & $2.54(0.05)$ & $0.03(-0.10$ to 0.15$)$ & 0.66 \\
\hline Well-being - enjoyment (1-4) & $2.73(0.05)$ & $2.66(0.06)$ & $0.07(-0.06$ to 0.19$)$ & 0.30 \\
\hline Well-being - control (1-4) & $2.55(0.05)$ & $2.61(0.05)$ & $-0.07(-0.19$ to 0.06$)$ & 0.27 \\
\hline
\end{tabular}

${ }^{a}$ Adjusted for the baseline score of the specific outcome variable, sex, age, marital status, educational level, and living arrangement

social and psychological functioning [38, 39]. Compared to other interventions, in which quality of life was considered an outcome variable, our study had a broader approach to frailty and therefore a different target group. In other interventions, older people were included in the interventions if they reported having problems [30, 31], visited the emergency department [27], were referred by family practitioners [34] or were screened by routine care data [16]. Accordingly, the differences in target groups between the interventions could possibly explain the difference in outcomes.

The WICM also had an effect on love and friendship, which are two important attributes of the quality of life of elderly [47]. Previous evaluation research on the short-term effects of the WICM also showed this effect [49], which indicates the consistency of this relevant finding. This consistent effect may be explained by the improved relationship between frail older people and their informal caregivers. In the WICM, the situation of frail older people is comprehensively assessed and monitored in consultation with the informal caregiver, possibly leading to tranquility and relief. This notion is underscored by the finding that the WICM had a positive effect on the subjective burden of the informal caregivers [50]. The informal caregivers indicated that their caregiver situation improved in terms of, for example, mental health and relationships, which could have affected the feelings of love and friendship experienced by frail older people.

Furthermore, the WICM did not show effects on health outcomes and functional abilities. Integrated care interventions such as the WICM, encompass the reorganization of care processes targeting at multi-dimensional needs of persons with similar problems [51]. However, this does not provide insight in the specific content of these care processes. Reorganization of care for frail older people might not be sufficient to achieve effectiveness in terms of health outcomes and functional abilities. The content of care might also be important; research has shown that integrated care containing specific medical and paramedical interventions has resulted in positive outcomes for frail older people $[29,52]$. With respect to medical and paramedical care, the differences between WICM and care as usual were limited, given that the Netherlands has a strong primary care system. An important distinction between WICM and care as usual is the multidisciplinary focus. The care in WICM is not purely medical but also entails prevention, residence and wellbeing. WICM's primary outcome measure was, therefore, quality of life [37].

\section{Strengths}

The strength of our study was its consideration of many different outcomes, which were measured with innovative instruments such as the ICECAP. The ICECAP instrument has been developed to measure older people well-being, even when personal functioning is not improving [53]. This instrument covers the five most important attributes of older adults' well-being, including love and friendship [46]. The effectiveness of integrated care has not been examined previously with this specific instrument. However, the ICECAP has been used in economic evaluations, in which it was shown that this instrument is more sensitive at detecting the effectiveness 
of interventions for frail older people than the EQ-5Dinstrument, a more traditional instrument to measure health-related quality of life [54].

\section{Limitations}

The primary limitation of our research is that the design of the study was quasi-experimental. To ensure that frail older people could receive care from their own GP, randomization of the frail older people population was not desirable. Our quasi-experimental design, however, means that the study population in the experimental and control groups could have differed non-randomly at baseline. In our study, the experimental group consisted of more women, more individuals living in assisted living facilities and more individuals with a lower level of education. However, these differences may not have impacted our results for two reasons. First, we accounted for these differences by including the background characteristics as covariates in our analyses. In these analyses, no significant effects were found for sex, living situation and educational level. Second, previous research has not shown consistent effects of these variables on factors such as quality of life $[55,56]$.

A second limitation is our focus on patient outcomes. Even though a comprehensive set of outcome measures was used in terms of health, functional abilities and quality of life, the effects of WICM on health care utilization remain to be determined. Integrated care has been shown to result in a decline in hospitalization and institutionalization [25]. Therefore, it would be useful to explore whether our integrated and pro-active intervention would affect health care utilization and associated costs. These costs could be compared with the effects of our intervention, such as health-related quality of life, to allow for statements regarding the cost-effectiveness of the WICM.

\section{Recommendations}

Recommendations for practice are that more in-depth insights into the effectiveness of preventive and integrated care approaches for frail older patients are required. Integrated care interventions such as the WICM should be further optimized in practice; it still remains unclear what specific combinations of pro-active and integrated care elements are most effective. The comprehensive WICM pursuing integration at the micro- and meso-level with a preventive focus showed moderate positive results in terms of quality of life but this intervention was not able to improve health outcomes and functional abilities. Furthermore, our study revealed that the specific content of care within these integrated care interventions for community-dwelling elderly should be carefully considered in the future development of these interventions including the WICM.
Regarding the outcomes for frail older people, future research is recommended to explore what specific outcomes could be expected for frail older people and how these outcomes could be accurately detected in evaluation research. Frailty is a gradual process of deterioration [4], and it might not be realistic to expect improvement or even preservation in all three domains (i.e., health, functional abilities and quality of life). However, our study shows that a slightly different emphasis, for example, by examining specific domains of wellbeing, is encouraging. In particular, the ICECAP instrument is recommended for inclusion in future evaluation research.

The final implication of this study for future research is enhancement of our understanding of the participants of integrated care interventions. Although all participating older people in the various studies have been described as frail, inclusion criteria or screening instruments to detect frailty in these studies were different (see also [25]). In addition, thus far, frail older people have been perceived as a single group in classical evaluation studies; no distinction of any kind has been made among frail older people, even though research has shown that they are a heterogeneous group of people with diverse problems in physical, psychological and social domains [4]. This heterogeneity should also be considered in the evaluation of integrated care and may possibly yield insight into its effectiveness.

\section{Conclusions}

The conclusion is that WICM, a pro-active and integrated care intervention with the GP-practice as single entry point, is moderately effective for communitydwelling frail older people. WICM had a positive effect on the ability to receive love and friendship and moderately preserves general quality of life; two relevant findings because they comprise the personal evaluation of the frail older people themselves. However, WICM was not effective in terms of health outcomes and functional abilities.

\section{Abbreviations \\ GFI: Groningen Frailty Indicator; GP: general practitioner; WICM: Walcheren Integrated Care Model.}

\section{Competing interests}

The authors declare that they have no competing interests.

\section{Authors' contributions}

WL collected the data, performed the statistical analyses and wrote the paper. IF was project leader of the study, planned the study, supervised the data collection and analyses and contributed to revising the paper. RK was one of the main initiators of the Walcheren Integrated Care Model and was responsible for its design and ensuring commitment of all parties involved. $\mathrm{RH}$ contributed to revising the paper. All authors have approved the submitted version of the manuscript and accept responsibility for the data presented. 


\section{Acknowledgments}

The implementation and evaluation of the WICM were part of the National Care for the Elderly Program (NPO), which is funded by the Netherlands Organization for Health Research and Development (ZonMW; project number 313030201). Part of the implementation of the model is also financed by the health insurer $C Z$. The authors acknowledge all health care professionals in Walcheren contributing to the implementation of the WICM.

\section{Received: 22 December 2015 Accepted: 1 February 2016}

\section{Published online: 15 February 2016}

\section{References}

1. WHO. Global health and Ageing. National Institute on Aging, National Institutes of Health. 2011. NIH publication no. 11-7737.

2. Friedman SM, Steinwachs DM, Rathouz PJ, Burton LC, Mukamel DB. Characteristics Predicting Nursing Home Admission in the Program of All-Inclusive Care for Elderly People. Gerontologist. 2005;45:157-66.

3. Wiles JL, Leibing A, Guberman N, Reeve J, Allen RES. The Meaning of "Ageing in Place" to Older People. Gerontologist. 2011;52:357-66.

4. Gobbens RJJ, Luijkx KG, Wijnen-Sponselee MTH, Schols JMGA. Towards a conceptual definition of frail community-dwelling older people. Nurs Outlook. 2010:58(2):76-86.

5. Schers HJ, Koopmans R, Olde RM. De rol van de huisarts bij kwetsbare ouderen. Een internetenquête onder huisartsen [The role of the GP for frail elderly. An internet survey among GPs]. Huisarts Wet. 2009;52(13):626-30.

6. Vedel I, De Stampa M, Bergman H, Ankri J, Cassou B, Blanchard F, et al. Healthcare professionals and managers' participation in developing an intervention: A pre-intervention study in the elderly care context. Implement Sci. 2009;4:21.

7. Kodner D. All together now: a conceptual exploration of integrated care. Healthc Q. 2009:13:6-15.

8. Boeckxstaens P, de Graaf P. Primary care and care for older persons: Position Paper of the European Forum for Primary Care. Qual Prim Care. 2011;19:369-89.

9. Fried LP, Tangen CM, Walston J, Newman AB, Hirsch C, Gottdiener J, et al. Frailty in older adults: Evidence for a phenotype. J Gerontol Med Sci. 2001; 56:M146-56.

10. Gobbens RJJ, van Assen MALM. The prediction of quality of life by physical, psychological and social components of frailty in community-dwelling older people. Qual Life Res. 2014;23:2289-300.

11. Challis D, Chessum R, Chesterman J, Luckett R, Woods B. Community Care for the Frail Elderly: An Urban Experiment. Br J Soc Work. 1987;18((supp):13-42.

12. Strandberg TE, Pitkälä KH. Frailty in elderly people. Lancet. 2007;369:1328-9.

13. Ex C, Gorter K, Janssen U. Providing integrated health and social care for older persons in the Netherlands. National report. Utrecht: Verwey Jonker Institute; 2003.

14. Gröne O, Garcia-Barbero M. Integrated care: a position paper of the WHO European Office for Integrated Health Care Services. International Journal of Integrated Care. 2001;1:e21.

15. Kodner DL, Kyriacou CK. Fully integrated care for frail elderly: two American models. International Journal of Integrated care. 2000;1, e08.

16. Bleijenberg N, Drubbel I, Schuurmans MJ, ten Dam VH, Zuithoff NPA, Numans ME, et al. The effectiveness of a proactive patient-centred primary care program on daily functioning of frail older patients: a cluster randomised controlled trial. In: Drubbel I, editor. Frailty screening in older patients in primary care using routine care data. Zutphen: CPI Koninklijke Wöhrmann BV; 2014. p. 125-54.

17. Johri $\mathrm{M}$, Beland $\mathrm{F}$, Bergman $\mathrm{H}$. International experiments in integrated care for the elderly: a synthesis of evidence. Int J Geriatr Psychiatry. 2003;18(3):222-35.

18. Fabbricotti IN. Zorgen voor zorgketens: integratie en fragmentatie in de ontwikkeling van zorgketens. [Taking care of integrated care: integration and fragmentation in the development of integrated care arrangements]. Rotterdam: Optima; 2007

19. Hébert R, Durand PJ, Dubuc N, Tourigny A, The PRISMA Group. PRISMA: a new model of integrated service delivery for the frail older people in Canada. International Journal of Integrated Care. 2003;3, e08.

20. Ross S, Curry N, Goodwin N. Case management. What it is and how it can best be implemented. London: The King's Fund; 2011. http://www. kingsfund.org.uk/sites/files/kf/Case-Management-paper-The-Kings-FundPaper-November-2011_0.pdf. Accessed 6 June 2015.
21. Kodner D, Spreeuwenberg C. Integrated care: meaning, logic, applications, and implications - a discussion paper. International Journal of Integrated Care. 2002;2:e12.

22. Béland F, Bergman H, Lebel P, Clarfield AM, Tousignant P, Contandriopoulos A-P, et al. A system of integrated care for older persons with disabilities in Canada: results from a randomized controlled trial. J Gerontol Med Sci. 2006;61A:367-73.

23. Bernabei R, Landi F, Gambassi G, Sgadari A, Zuccala G, Mor V, et al. Randomised trial of impact of model of integrated care and case management for older people living in the community. BMJ. 1998;316: $1348-51$

24. Chatterji P, Burstein NR, Kidder D, White A. Evaluation of the Program of AllInclusive Care for the Elderly (PACE) Demonstration. The Impact of PACE on Participant Outcomes. 1998. https://www.cms.gov/Medicare/DemonstrationProjects/DemoProjectsEvalRpts/downloads/pace_outcomes.pdf. Accessed 8 June 2015.

25. Eklund K, Wilhelmson K. Outcomes of coordinated and integrated interventions targeting frail elderly people: a systematic review of randomized controlled trails. Health Soc Care Community. 2009:17(5):447-58.

26. Eklund K, Wilhelmson K, Gustafsson H, Landahl S, Dahlin-Ivanoff S. One-year outcome of frailty indicators and activities of daily living following the randomised controlled trial; "Continuum of care for frail older people". BMC Geriatr. 2013;13:76

27. Gagnon AJ, Schein C, McVey L, Bergman H. Randomized controlled trial of nurse case management of frail older people. J Am Geriatr Soc. 1999;47: $1118-24$

28. Hébert R, Raîche M, Dubois M-F, Gueye NR, Dubuc N, Tousignant M, et al. Impact of PRISMA, a coordination-type integrated service delivery system for frail older people in Quebec (Canada): a quasi-experimental study. J Gerontol Soc Sci. 2010:65B(1):107-18

29. Leveille SG, Wagner EH, Davis C, Grothaus L, Wallace J, LoGerfo M, et al. Preventing disability and managing chronic illness in frail older adults: a randomized trial of a community-based partnership with primary care. J Am Geriatr Soc. 1998:46:1191-8.

30. Markle-Reid M, Weir R, Browne G, Roberts J, Gafni A, Henderson S. Health promotion for frail older home care clients. J Adv Nurs. 2006;54:381-95.

31. Melis RJ, van Eijken MIJ, Teerenstra S, van Achterberg T, Parker SG, Borm GF, et al. A randomized study of a multidisciplinary program to intervene on geriatric syndromes in vulnerable older people who live at home (Dutch EASYcare Study). J Gerontol Med Sci. 2008;63A(3):283-90.

32. Metzelthin SF, Van Rossum E, De Witte LP, Ambergen T, Sipers W, Hobma S, et al. Effectiveness of an interdisciplinary primary care approach to reduce disability in community-dwelling frail older people: a cluster randomised controlled trial. BMJ. 2013;347:f5264.

33. Montgomery P, Fallis W. South Winnipeg Integrated Geriatric Program (SWING): a rapid-community response program for the frail elderly. Can J Aging. 2003;22:275-81.

34. Rockwood K, Stadnyk K, Carver D, MacPherson KM, Beanlands HE, Powell C, et al. A clinimetric evaluation of specialized geriatric care for rural dwelling, frail older people. J Am Geriatr Soc. 2000;48:1080-5.

35. Tourigny A, Durand PJ, Bonin L, Hébert R, Rouchette L. Quasi-experimenta Study of the Effectiveness of an Integrated Service Delivery Network for the Frail Elderly. Can J Aging. 2004:23(3):229-43.

36. Van Hout HPJ, Jansen APD, van Marwijk HWJ, Pronk M, Frijters DF, Nijpels G. Prevention of Adverse Health Trajectories in a Vulnerable Elderly Population Through Nurse Home Visits: A Randomized Controlled Trial. J Gerontol Med Sci. 2010;65(7):734-42

37. Fabbricotti IN, Janse B, Looman WM, de Kuijper R, van Wijngaarden JDH, Reiffers A. Integrated care for frail elderly compared to usual care: a study protocol of a quasi-experiment on the effects on the frail elderly, their caregivers, health professionals and health care costs. BMC Geriatr. 2013;13:31.

38. Peters $L L$, Boter H, Buskens E, Slaets JPJ. Measurement properties of the Groningen Frailty Indicator in home-dwelling and institutionalized elderly people. J Am Med Dir Assoc. 2012:13(6):546-51.

39. Schuurmans H, Steverink N, Lindenberg S, Frieswijk N, Slaets JPJ. Old or frail: what tells us more? J Gerontol Med Sci. 2004:59A:962-5.

40. Boot JM, Knapen MJMH. Handboek Nederlandse gezondheidszorg. [Handbook Dutch Healthcare]. Utrecht: Het Spectrum; 2005.

41. Smelt AFH, van der Weele GM, Blom JW, Gussekloo J, Assendelft WJ. How usual is usual care in pragmatic intervention studies in primary care? An overview of recent trials. Br J Gen Pract. 2010;60(576):e305-18. 
42. Van Der Zee Kl, Sanderman R. Het meten van de algemene gezondheidstoestand met de RAND-36: Een handleiding [The assessment of general health status with the RAND-36]. Groningen: Research Institute SHARE/University of Groningen, the Netherlands; 2012.

43. Laan W, Zuithoff NPA, Drubbel I, Bleijenberg N, Numans ME, de Wit NJ, et al. Validity and reliability of the Katz-15 scale of measure unfavorable health outcomes in community-dwelling older people. J Nutr Health Aging. 2014; 18(9):848-54.

44. Krabbe PF, Stouthard ME, Essink-Bot ML, Bonsel GJ. The effect of adding a cognitive dimension to the EuroQol multiattribute health-status classification system. J Clin Epidemiol. 1999:52(4):293-301.

45. Lamers LM, McDonnell J, Stalmeier PFM, Krabbe PFM, Busschbach JJV. The Dutch tariff: Results and arguments for an effective design for national EQ5D valuation studies. Health Econ. 2006;15(10):1121-32.

46. Coast J, Flynn TN, Natarajan L, Sproston K, Lewis J, Louviere JL, et al. Valuing the ICECAP capability index for older people. Soc Sci Med. 2008;67:874-82.

47. Grewal I, Lewis J, Flynn TN, Brown J, Bond J, Coast J. Developing attributes for a generic quality of life measure for older people: preferences or capabilities? Soc Sci Med. 2006;62:1891-901.

48. Cohen J. Statistical power analysis for the behavioral sciences. 2nd ed. Hillsdale, New Jersey: Lawrence Erlbaum Associates; 1988.

49. Looman WM, Fabbricotti IN, Huijsman R. The short-term effects of an integrated care model for the frail elderly on health, quality of life, health care use and satisfaction with care. International Journal of Integrated Care. 2014; 14, e034.

50. Janse B, Huijsman R, Kuyper RDM, Fabbricotti IN. The effects of an integrated care intervention for the frail elderly on informal caregivers: a quasi-experimental design. BMC Geriatr. 2014;14:58

51. Nies H. Integrated Care: concepts and background. In: Nies H, Berman PC, editors. Integrating Services for Older People: a resource book for managers. Dublin: Ehma; 2004. p. 17-32.

52. Gill TM, Baker DI, Gottschalk M, Peduzzi PN, Allore H, Byers A. A program to prevent functional decline in physically frail, elderly persons who live at home. N Engl J Med. 2002;347(14):1068-74.

53. Makai P, Brouwer WB, Koopmanschap MA, Nieboer AP. Capabilities and quality of life in Dutch psycho-geriatric nursing homes: an exploratory study using a proxy version of the ICECAP- O. Qual Life Res. 2012;21(5):801-12.

54. Makai P, Looman WM, Adang E, Melis R, Stolk E, Fabbricotti IN. Costeffectiveness of integrated care in frail elderly using the ICECAP-O and EQ5D: does choice of instrument matter? Eur J Health Econ. 2015;16:437-50.

55. Lee TW, Ko IS, Lee KJ. Health promotion behaviors and quality of life among community-dwelling elderly in Korea: A cross-sectional survey. Int J Nurs Stud. 2006;43(3):293-300.

56. Grayson P, Lubin B, Van Whitlock R. Comparison of depression in the community-dwelling and assisted-living elderly. J Clin Psychol. 1995;51(1):19-21.

\section{Submit your next manuscript to BioMed Central and we will help you at every step:}

- We accept pre-submission inquiries

- Our selector tool helps you to find the most relevant journal

- We provide round the clock customer support

- Convenient online submission

- Thorough peer review

- Inclusion in PubMed and all major indexing services

- Maximum visibility for your research

Submit your manuscript at www.biomedcentral.com/submit
O BioMed Central 